\title{
PIV of air flow over a step and discussion of fluctuation decompositions
}

Daniel Duda, and Václav Uruba

Citation: AIP Conference Proceedings 2000, 020005 (2018); doi: 10.1063/1.5049912

View online: https://doi.org/10.1063/1.5049912

View Table of Contents: http://aip.scitation.org/toc/apc/2000/1

Published by the American Institute of Physics

\section{Articles you may be interested in}

Preface: $37^{\text {th }}$ Meeting of Departments of Fluid Mechanics and Thermodynamics

AIP Conference Proceedings 2000, 010001 (2018); 10.1063/1.5049906

Mixed convection heat and mass transfer in horizontal channel heated from below: Radiation influence

AIP Conference Proceedings 2000, 020004 (2018); 10.1063/1.5049911

On Reynolds number physical interpretation

AIP Conference Proceedings 2000, 020019 (2018); 10.1063/1.5049926

Dependance of saturated water steam consumption on the moisture content of black locust sawn timber during the colour modification process

AIP Conference Proceedings 2000, 020006 (2018); 10.1063/1.5049913

The use of the Peltier modules for the thermal management of a metal hybride tank

AIP Conference Proceedings 2000, 020002 (2018); 10.1063/1.5049909

Impact of exhaust gas recirculation on production of nitrogen oxides in natural gas combustion

AIP Conference Proceedings 2000, 020003 (2018); 10.1063/1.5049910

\section{Conference Proceedings}

\section{Get $30 \%$ off all \\ Enter Promotion Code $P D=30$ at checkout print proceedings!}




\title{
PIV of Air Flow over a Step and Discussion of Fluctuation Decompositions
}

\author{
Daniel Duda ${ }^{1, a)}$ and Václav Uruba ${ }^{2, b)}$ \\ ${ }^{1}$ University of West Bohemia in Pilsen, Univerzitni 22, Pilsen, Czech Republic \\ ${ }^{2}$ Institute of Thermomechanics, Czech Academy of Sciences, Dolejškova 5, Prague, Czech Republic \\ a)dudad@kke.zcu.cz \\ b) uruba@it.cas.cz
}

\begin{abstract}
The experimental method of PIV (Particle image velocimetry) is used to study the air flow over a forward facing step creating shear layer between the outer flow and the recirculation bubble. This layer decays into vortices, which are highlighted by using Reynolds (temporal) decomposition or Agrawal (spatial) decomposition. The later can be tuned to different-sized structures, and, additionally, their energies roughly follow the Kolmogorov $-5 / 3$ law.
\end{abstract}

\section{INTRODUCTION}

The well-studied case of a flow over a forward-facing step is used as an example case for discuss the decomposition to a fluctuating velocity field. The fluctuating component is often ignored in both, experimental and numerical studies, but it contains the key component of turbulence - vortices and other coherent structures [1].

The approach to describing turbulence via individual coherent structures seems to be important especially in the light of recent discoveries in superfluid helium, which is a quantum liquid driven microscopically by totally different laws, but displaying the same macroscopic behavior as the classical fluids [2]. And, what are common for both, are the vortices.

\section{EXPERIMENTAL SETUP}

The geometry of the step and the studied fields of view (FoV) are depicted in Figure 1. FoV is located above a positive step of height $H=35 \mathrm{~mm}$ situated perpendicularly to the axis of the wind tunnel of square cross-section $125 \times 125 \mathrm{~mm}$. The air velocity $U=7.5 \mathrm{~m} / \mathrm{s}$, which corresponds to Reynolds number $R e=U \cdot H / v=1.8 \cdot 104$.

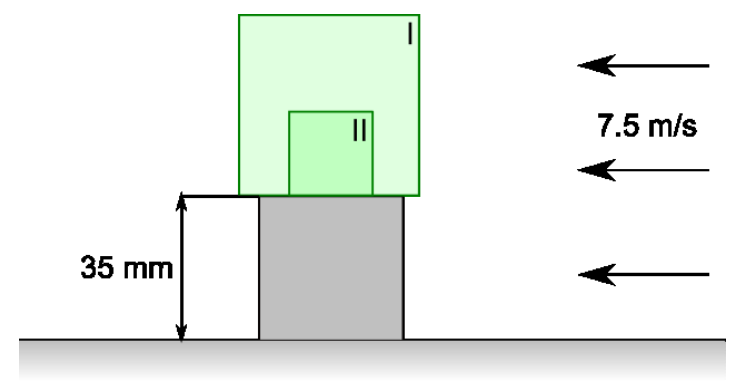

FIGURE 1. Sketch of the obstacle (gray) and field of view (light green) positioning. The size of field of view is $43.1 \times 43.1 \mathrm{~mm}$ for the case I and $20.5 \times 20.5 \mathrm{~mm}$ for the case II.

37th Meeting of Departments of Fluid Mechanics and Thermodynamics AIP Conf. Proc. 2000, 020005-1-020005-4; https://doi.org/10.1063/1.5049912 Published by AIP Publishing. 978-0-7354-1716-8/\$30.00 
The measurement method Particle Image Velocimetry (PIV) with double pulse timing [3] is used in air and with glycerin droplets. The size of the Interrogation Area (IA) in current study is $0.67 \mathrm{~mm}$ for the FoV I, and it is $0.32 \mathrm{~mm}$ for the smaller studied FoV II. The corresponding time between two consecutive frames is 50 or $25 \mu \mathrm{s}$ respectively. The PIV method averages the velocity under the scale of 1 IA, therefore, taking into account the noise and errors, the size of the smallest trustworthy structures is about 2 IA.

\section{MEAN VELOCITY FIELD}

The average over 100 samples of the velocity is shown in Figure 2. Together with the standard deviation $\operatorname{sd}(\mathbf{v})$, $\operatorname{sd}^{2}(\mathbf{v})=\left\langle\mathbf{v}^{2}\right\rangle-\langle\mathbf{v}\rangle^{2}$, where $\mathbf{v}$ is the velocity vector and $\langle\cdot\rangle$ means the time averaging. We can see that the plane of maximum fluctuations lies above the plane of zero velocity, which, in the mean flow field, separates the recirculation bubble. In individual realizations, the velocity is definitely not zero there; it is just the effect of averaging, as we can judge from one example shown in Figure 3.

The smaller field of view (referred as FoV II) is chosen in order to show the "wild" part of the flow field with already developed turbulent shear layer. Its size is the minimum possible with the current lens, because, due to symmetry, we prefer to keep the studied plane in the middle of the wind tunnel.

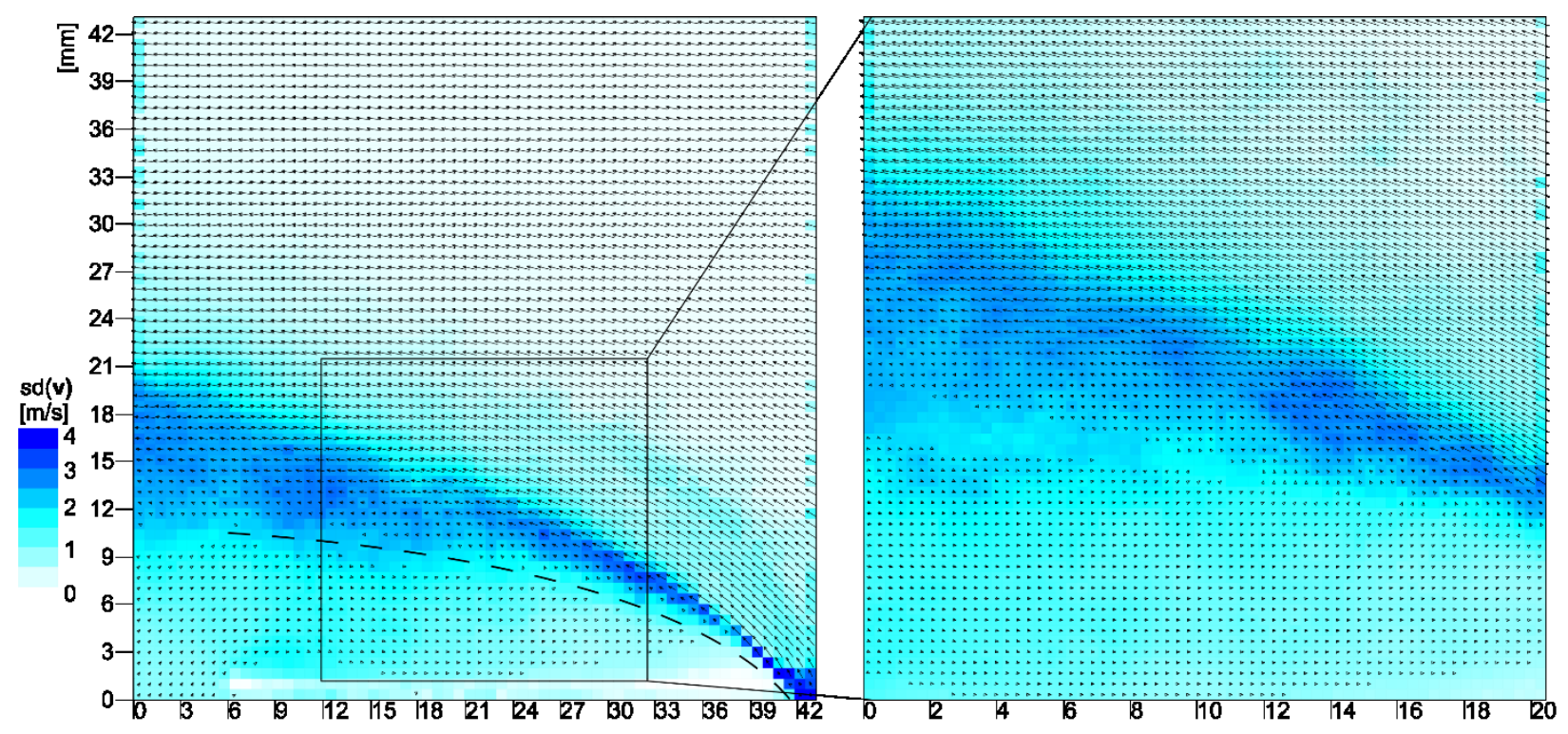

FIGURE 2. Average velocity field for both cases. The color corresponds to the standard deviation of local velocity vector calculated from the set of 100 realizations. The black square in the left figure shows the relative position of the second figure.

The dashed line in left Fig. highlights the position of zero velocity separating the recirculation bubble.

\section{FLUCTUATING VELOCITY FIELDS}

The time-fluctuating component $\mathbf{v}$ ' is obtained by the so-called Reynolds decomposition, which shows only that, what is different from the mean:

$$
\mathbf{v}^{\prime}(\mathbf{x}, t)=\mathbf{v}(\mathbf{x}, t)-\langle\mathbf{v}(\mathbf{x})\rangle_{T}
$$

The Reynolds decomposition assumes that the local advection velocity, which deforms the image of the vortex in the instantaneous velocity field $\mathbf{v}$, is equal to the local time-averaged velocity [4]. See the example in the left panel of Figure 3.

On the other hand, the spatial-fluctuating component $\mathbf{~}_{\text {Pas, }}$ also referred as the spatial or Agrawal decomposition, is calculated with using single instantaneous velocity field $\mathbf{v}$ as the convolution with the band-pass filter [5]:

$$
\mathbf{v}_{\text {Pas }}(\mathbf{x}, t)=\mathbf{v}(\mathbf{x}, t) * P(\mathbf{x})=\int \mathbf{v}(\mathbf{x}-\mathbf{y}, t) P(\mathbf{y}) \mathbf{d} \mathbf{y}
$$

where $P$ is the band-pass filter defined as a difference of two Gaussians $G$ with widths $\sigma_{H}$ and $\sigma_{L}$

$$
P(\mathbf{x})=G\left[\sigma_{L}\right](|\mathbf{x}|)-G\left[\sigma_{H}\right](|\mathbf{x}|)=\frac{1}{\sqrt{2 \pi}}\left(\frac{1}{\sigma_{L}} \mathrm{e}^{\mathbf{x}^{2} / 2 \sigma_{L}^{2}}-\frac{1}{\sigma_{H}} \mathrm{e}^{\mathbf{x}^{2} / 2 \sigma_{H}^{2}}\right) .
$$


The main assumption of the Agrawal decomposition is that the local advection velocity is equal to the mean velocity of the surroundings of size $\sigma_{H}$, which is determined by structures larger than $\sigma_{H}$. See one example in the right panel of Figure 3.
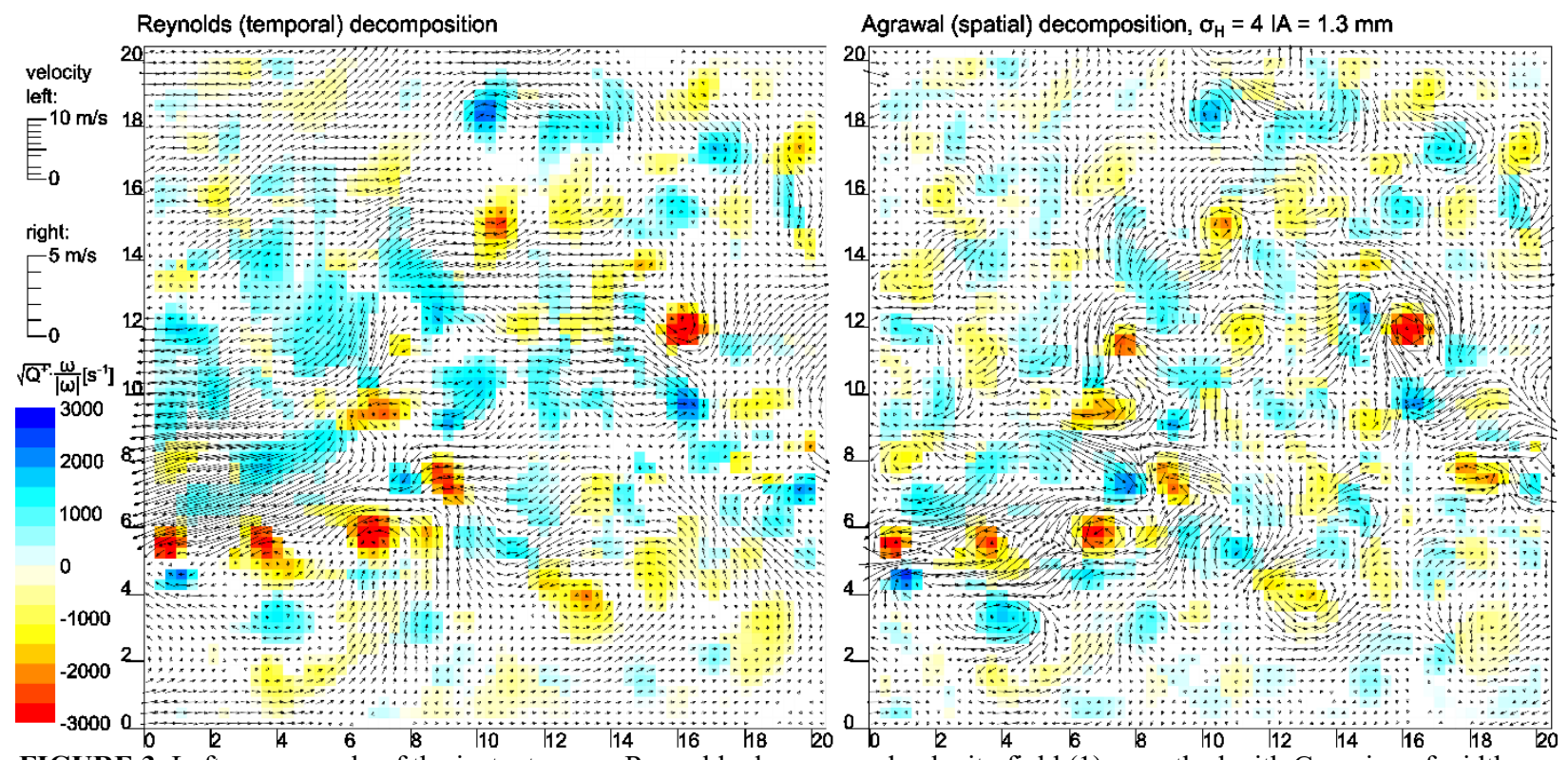

FIGURE 3. Left: an example of the instantaneous Reynolds-decomposed velocity field (1) smoothed with Gaussian of width $\sigma=$ $1 \mathrm{IA}=0.32 \mathrm{~mm}$. Right: the Agrawal decomposition (2) of the same realization with using $\sigma_{H}=1.3 \mathrm{~mm}$ and $\sigma_{L}=0.32 \mathrm{~mm}$.

Color corresponds to $q_{o r}$ defined in the text.

While the Reynolds decomposition subtracts also the structures, which repeat - e.g. the shear layer, the Agrawal decomposition removes the extensive uniformly moving masses of fluid, which are a type of coherent structures as well, and which carry significant part of energy of fluctuations. For example, individual $\sigma_{H}$-sized vortices are better visible by using the Agrawal decomposition, while the oscillations of entire shear layer can be studied in the temporal fluctuating velocity fields.

Important vortices are observable by using both approaches, which is depicted if Figure 3 by the oriented squareroot of $Q$ plotted in Figure 3 and defined as $q_{o r}=\left(Q^{+}\right)^{1 / 2} \cdot \operatorname{sign} \omega$, where $\omega$ is the vorticity and $Q^{+}$is the positive part of $Q$-invariant of the local velocity gradient tensor, $\mathrm{Q}=\left(\omega^{2}-s^{2}\right) / 2$ where $\mathrm{s}$ is the shear.

\section{ENERGY SPECTRUM}

The Agrawal decomposition typically describes less energy of the flow field, but it offers more manipulation space by selecting the scale of interest. The energy content of different scales of spatially fluctuating velocity field roughly follows the famous Kolmogorov law for homogenous isotropic turbulence although this case is turbulence in decaying shear layer, see Figure 4.

The range of scales is limited from one side by the size of one IA, from the other side by the appropriate fraction of the size of FoV (such, that the area influenced by boundary of FoV is smaller than the undisturbed area). This quite strong limitation causes that only a short part of cascade is visible.

The flattening at highest $k$ apparent in Figure 4 for both FoVs is probably caused by instrumental noise, which is believed to act at the smallest scale - 1 IA. By using the expectation, that the last point might continue with the Kolmogorov scaling, we can roughly evaluate the energetic contribution of the instrumental noise at the smallest scale to be $30 \%$ in FoV I and $25 \%$ in FoV II respectively. It is questionable, if the usage of theoretical exponent is adequate, when the observed slope of spectrum is apparently steeper, as can be judged form Figure 4. Anyway, this result suggests that the small scale noise usually observed in PIV results is still dominated by the real turbulent noise, although the instrumental one is relevant (at least in this case).

The standard methods of measuring energy spectra have to use the Taylor hypothesis of frozen turbulence, because they measure the time spectra in fact. Here we can obtain directly the space spectrum, ideally by using a 
single shot only - of course, the statistical quality would be poor in that case, as can be seen from errorbars in Figure 4.

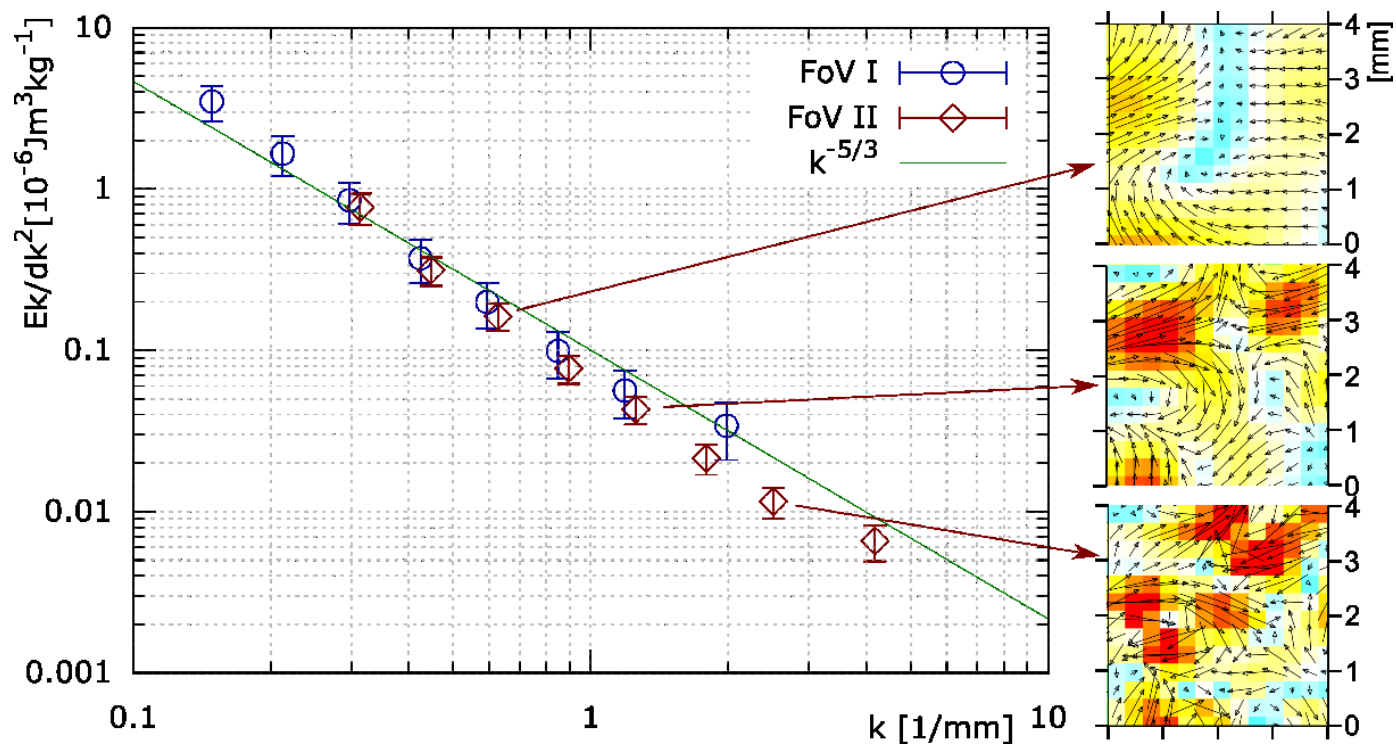

FIGURE 4. The energy content of the spatially decomposed velocity fields plotted as a function of the wave-number $k=2 /\left(\sigma_{H}+\sigma_{\mathrm{L}}\right)$. The rough energy content of each band is multiplied by $k$ and divided by square of the band width $d k=1 / \sigma_{L}-1 / \sigma_{\mathrm{H}}$. The errorbars are result of statistical scatter of 100 independent realizations. Right panels show three examples of marked velocity fields, all at the same space and time location; color corresponds to velocity magnitude - light blue: 0 , red: $0.4 \mathrm{~m} / \mathrm{s}$.

\section{CONCLUSION}

We compared two possible decompositions to fluctuating velocity component. The first one is the standard temporal decomposition referred as Reynolds decomposition, the second is a less used spatial Agrawal decomposition based on subtracting local large-scale structures, which allows us to focus to a specific-sized structures. This approach seems to be a key to decompose the velocity field into a set of individual vortices, which are overlapped hiding each other. Surprisingly, just the energies of different-width Agrawal decompositions without any vortex identification can follow the Kolmogorov spectrum of vortex cascade.

\section{ACKNOWLEDGMENTS}

This work was supported by the project SGS-2016-045 (Increase of efficiency, reliability and a lifespan of power machines and devices 4).

\section{REFERENCES}

1. V. Uruba, O. Hladík and P. Jonáš, J. Phys. Conf. Ser. 318, 062021 (2011).

2. M. La Mantia, P. Švančara, D. Duda and L. Skrbek, Phys. Rev. B 94, 184512 (2016).

3. V. Kopecký. Metody laserové anemometrie v experimentální mechanice tekutin, (Technická univerzita $\mathrm{v}$ Liberci, Liberec, 2001).

4. R. J. Adrian, K. T. Christensen and Z. C. Liu, Exp. Fluids 29, 275-290 (2000).

5. A. Agrawal and A. Prasad, Exp. Fluids 33, 565-577 (2002). 